\title{
Human-Robot Physical Interaction with Dynamically Stable Mobile Robots
}

\author{
Umashankar Nagarajan \\ The Robotics Institute \\ Carnegie Mellon University \\ Pittsburgh, PA, USA \\ umashankar@cmu.edu
}

\author{
George Kantor \\ The Robotics Institute \\ Carnegie Mellon University \\ Pittsburgh, PA, USA \\ kantor@ri.cmu.edu
}

\author{
Ralph L. Hollis \\ The Robotics Institute \\ Carnegie Mellon University \\ Pittsburgh, PA, USA \\ rhollis@cs.cmu.edu
}

\begin{abstract}
Human-Robot Physical Interaction is an important attribute for robots operating in human environments. The authors illustrate some basic physically interactive behaviors with dynamically stable mobile robots using the ballbot as an example. The ballbot is a dynamically stable mobile robot moving on a single spherical wheel. The dynamic stability and robust controllers enable the ballbot to be physically moved with ease. The authors also demonstrate other behaviors like human intent detection and learn-repeat behavior on the real robot.
\end{abstract}

Categories and Subject Descriptors: J.2 [Physical Sciences and Engineering]: Engineering, Physics

General Terms: Experimentation, Human Factors.

Keywords: Human-Robot Physical Interaction, Dynamically Stable Mobile Robots.

\section{INTRODUCTION}

The ballbot, introduced in [1], is a dynamically stable single spherical wheeled mobile robot capable of omnidirectional motion. Unlike statically stable mobile robots, dynamically stable mobile robots can be tall and skinny with high centers of gravity and small bases ([2]). These features make dynamically stable mobile robots more suitable for navigation and interaction in human environments.

Humans are physically interactive with everything in their environment. Therefore, any robot that would be a part of this environment needs to handle physical interactions with humans and objects ([3]). The dynamic stability in robots like the ballbot naturally enable various physically interactive behaviors, some of which are described in the sections below.

\section{THE BALLBOT}

The ballbot uses a four-motor inverse mouse-ball drive unit for actuating the ball. The ballbot has an inertial measurement unit (IMU) that provides information on the body

Copyright is held by the author/owner(s). HRI'09, March 11-13, 2009, La Jolla, California, USA. ACM 978-1-60558-404-1/09/03.

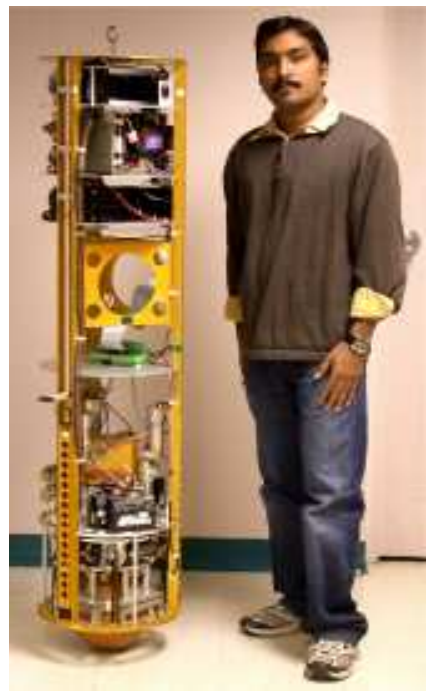

Figure 1: Ballbot balancing.

angles and angular rates with respect to gravity. It also has a yaw drive mechanism, which enables yaw rotation of the body about its vertical axis. There are three legs that provide static stability to the ballbot when powered down.

The balancing controller on the ballbot is designed to keep the center of the ball right below the center of mass of the body. There are various controllers in the ballbot that enable it to station-keep, track trajectories on the floor and come to a stop when given a large disturbance. The ballbot can also be moved around using a joystick controller. The ballbot is capable of auto-transitioning from a statically stable state with the three legs fully down to a dynamically stable state with the three legs retracted onto the body. Details of the controllers can be found in [4].

\section{HUMAN-ROBOT PHYSICAL INTERAC- TION}

Physical interactions between humans and robots play an important role while operating in human environments. The authors attempt to demonstrate human-robot physical interaction with dynamically stable mobile robots using the ballbot as an example.

\subsection{Ease of Mobility}

While operating in human environments, the human user should be able to physically move the robot with ease. While 
balancing, the ballbot can be moved around with very little effort. Physically directing a heavy statically stable mobile robot can be a difficult task, whereas the ballbot can be moved around with just a single finger (Figure 2). Similarly, while in motion, robots should be physically stoppable and controllable by a human with minimal force. Even while moving, the ballbot can be stopped with just a single finger. The ballbot also has a passive lever hand that can be used to drag it around. Just like you would take a dog for a walk, you can take the ballbot for a roll. This ease of mobility is a courtesy of dynamic stability.

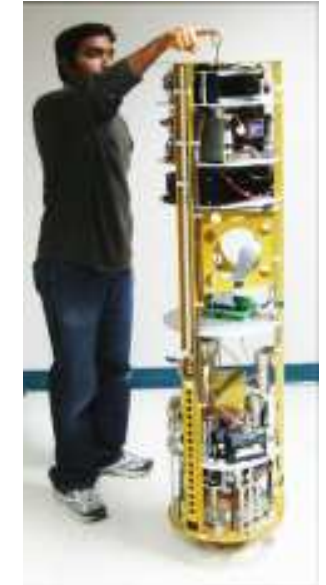

(a) With a finger

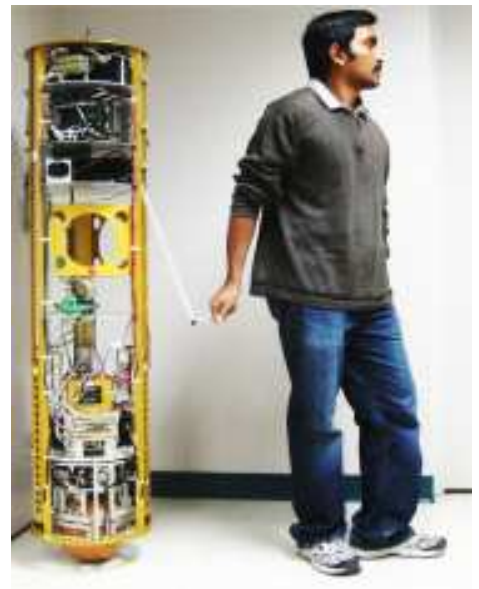

(b) With a passive lever hand
Figure 2: Moving the ballbot

\subsection{Robustness}

While operating in cluttered human environments, robots should be robust to handle user mistakes and even a certain degree of ill-treatment. They have to be robust to disturbances. The balancing controller on the ballbot is robust to large disturbances like even shoves and kicks. It is also robust to collisions with furniture and walls.

\subsection{Human Intent Detection}

Basic human intentions can be detected using the amount of force applied by the human user on the robot. For example, the ballbot continues to station-keep when subjected to small disturbances like a soft push whereas, given a hard push, it moves away from its current location and stationkeeps at a different point on the floor as shown in Figure 3.

\subsection{Learn and Repeat}

Physical interaction can be used to teach various tasks to the robot. The authors present the initial results in developing a Learn-Repeat behavior in the ballbot. In the learn mode, the user moves the ballbot in a desired path and in the repeat mode, the ballbot attempts to track the learnt path. Attempts at repeating approximate linear and circular paths learnt from the human user are shown in Figure 4.

\section{FUTURE WORK}

All the above illustrated behaviors were achieved using just the IMU and ball motor encoders. The authors are in the process of adding laser range finders and stereo cameras

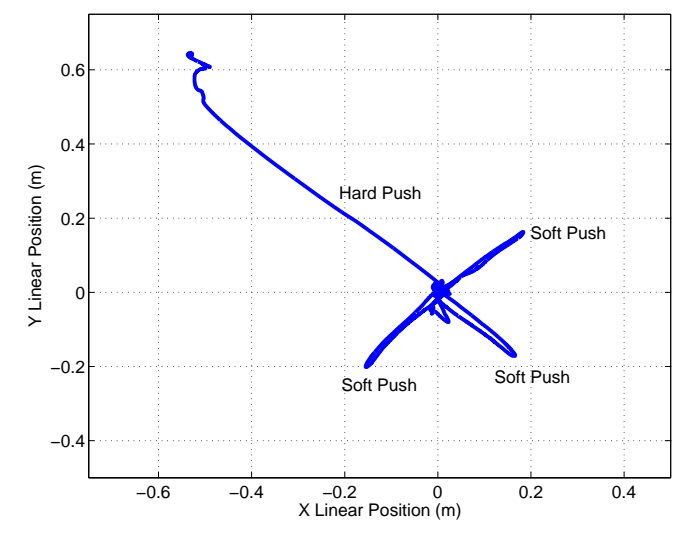

Figure 3: Human Intent Detection - XY Plot

that would enable better localization. These sensors would enable the authors to explore more aspects of Human-Robot Interaction.

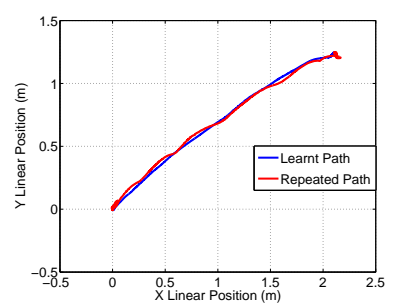

(a) Linear Motion

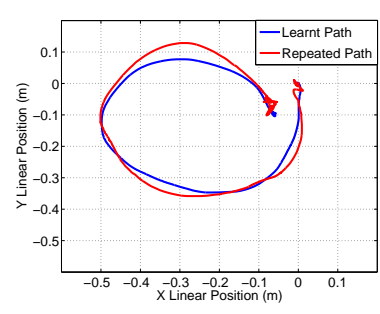

(b) Circular Motion
Figure 4: Learn-Repeat behavior in the ballbot

\section{ACKNOWLEDGMENTS}

This work is funded in part by NSF Grants IIS-0308067 and IIS-0535183.

\section{REFERENCES}

[1] T. B. Lauwers, G. A. Kantor, and R. L. Hollis. A dynamically stable single-wheeled mobile robot with inverse mouse-ball drive. In Proc. Int'l. Conf. on Robotics and Automation, October 12-15 2005.

[2] R. Hollis. Ballbots. Scientific American, pages 50-55, October 2006.

[3] R. Alami et al. Safe and dependable physical humanrobot interaction in anthropic domains: State of the art and challenges. In Workshop on Physical Human-Robot Interaction in Anthropic Domains, IEEE/RSJ International Conference on Intelligent Robots and Systems, October 9-12 2006.

[4] U. Nagarajan, A. Mampetta, G. Kantor, and R. Hollis. State transition, balancing, station keeping, and yaw control for a dynamically stable single spherical wheel mobile robot. In Int'l. Conf. on Robotics and Automation (Accepted), May 12-17 2009. 